International Journal of Applied Dental Sciences 2021; 7(4): 317-321

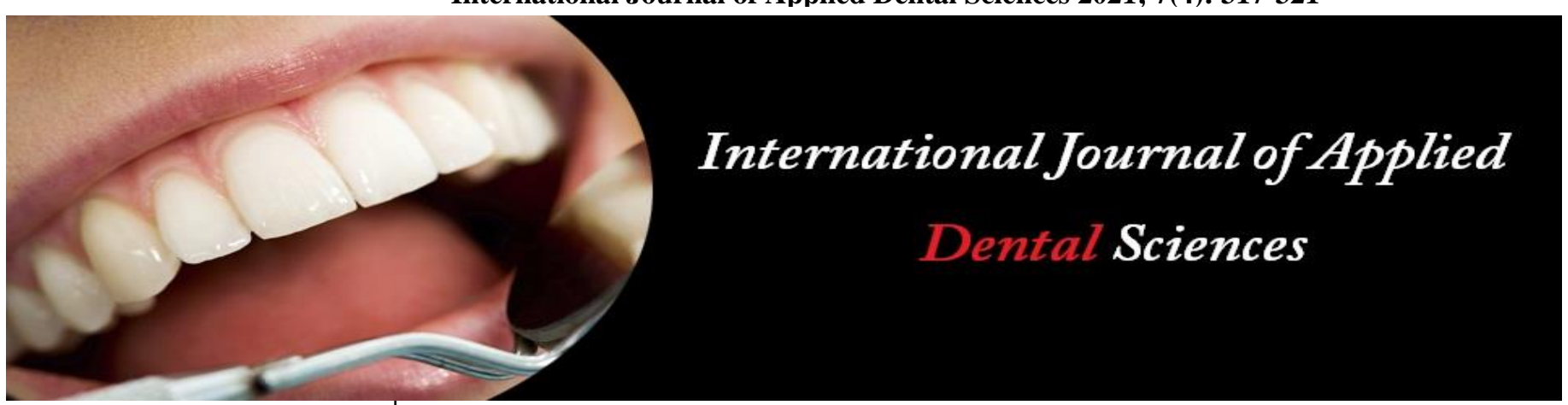

ISSN Print: 2394-7489

ISSN Online: 2394-7497

IJADS 2021; 7(4): 317-321

(C) 2021 IJADS

www.oraljournal.com

Received: 19-08-2021

Accepted: 21-09-2021

Shubham Kajani

Post Graduate Student,

Department of Conservative Dentistry and Endodontics,

Bapuji Dental College and

Hospital, Davangere,

Karnataka, India

Sophia Thakur

Professor, Department of

Conservative dentistry and

Endodontics, Bapuji Dental

College and Hospital, Davangere,

Karnataka, India

Arun Jayasheel

Reader, Department of

Conservative Dentistry and

Endodontics, Bapuji Dental

College and Hospital, Davangere,

Karnataka, India
Corresponding Author:

Shubham Kajani

Post Graduate Student,

Department of Conservative

Dentistry and Endodontics,

Bapuji Dental College and

Hospital, Davangere,

Karnataka, India

\section{An expeditious update on single visit endodontic retreatment: A case series}

\author{
Shubham Kajani, Sophia Thakur and Arun Jayasheel
}

DOI: $\underline{\text { https://doi.org/10.22271/oral.2021.v7.i4e.1389 }}$

\section{Abstract}

Adequate cleaning and shaping of the canals to reduce the microorganisms below the threshold level is one of the most important goals of endodontic treatment. With the advent of newer materials and techniques, this goal can be achieved in a single appointment in many teeth which have been endodontically treated, but have failed the endodontic treatment. This case series highlights single visit endodontic retreatment as an important aspect of day-to-day dental practise.

Keywords: endodontic retreatment, single-visit, periapical index

\section{Introduction}

With the introduction of novel cleaning and shaping techniques and materials, root canal treatments can be completed safely in a single visit instead of multiple visits. Root canal retreatment is a type of procedure that is applied when previous RCTs have failed ${ }^{[1]}$. Postoperative pain after endodontic retreatment is an undesirable occurrence for patients and clinicians ${ }^{[2]}$.

The orthograde retreatment of dental elements previously treated with the most varied techniques is a fairly common clinical practice, particularly for endodontic specialists ${ }^{[3]}$. The decision-making process should consider the many different variables such as presence or absence of apical periodontitis, degree of pre-operative pain and the Periapical Index (PAI) ${ }^{[4]}$. Completing the treatment in a single appointment reduces the treatment time and cost, decreases microleakage risk and avoids recontamination of root canals between appointments in multiple-visit treatments ${ }^{[4]}$. In fact, no significant differences were observed in healing of periapical radiolucency between teeth treated in one visit (without) and those treated in two visits using calcium hydroxide for 4 weeks and that the presence of a positive bacterial culture at the time of filling did not influence the outcome of treatments ${ }^{[5]}$.

This case series highlights the need for practising "single visit endodontic retreatments" on a daily basis and the considerations to be acknowledged while performing them.

\section{Case Report 1}

A 34-year-old female patient reported to the Department of Conservative Dentistry and Endodontics with a chief complaint of pain in the upper left back tooth region in the last 9 days. While recording case history, she revealed that she had got an endodontic treatment done in the same region 2 months ago. On clinical examination, temporary restorative material was noted in relation to the tooth 27 . On radiographical examination, it was revealed that the root canal fillings were $5 \mathrm{~mm}$ beyond the apices of the three roots. (Fig 1a) The pain was moderate, intermittent and aggravated on chewing food with no history of swelling. The patient had to travel a long distance back after the treatment. Considering these clinical factors, single-visit endodontic retreatment procedure was initiated for the tooth 27 . The temporary restoration removal was followed by removal of gutta percha using Pro-Taper Retreatment files and Hfiles. (Figs 1b, 1c) No chemical solvents were used to remove the gutta-percha or the sealer. The working length was determined after confirming apical patency with \#15 K-file, and cleaning and shaping was done using Pro-Taper GOLD rotary files, and irrigation was done with $2.5 \%$ sodium hypochlorite activated using Endoactivator. 
This was followed by use of $17 \%$ EDTA and saline rinse. Final rinse was done using 2\% Chlorhexidine. The master cone IOPA was followed after confirming with the tug back. (Fig 1d) The obturation was done using cold lateral condensation method followed by post-endodontic composite restoration. (Figs 1e, 1f) The 3-month follow-up radiograph (Fig 1g) is shown, and clinically reduction of the symptoms indicates successful endodontic retreatment.

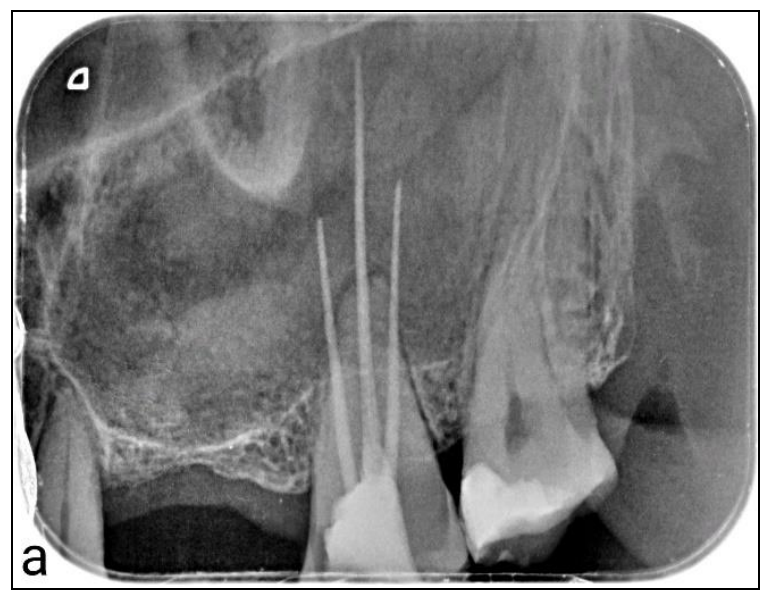

Fig 1a: Pre-operative radiograph (gutta percha $5 \mathrm{~mm}$ beyond apex)

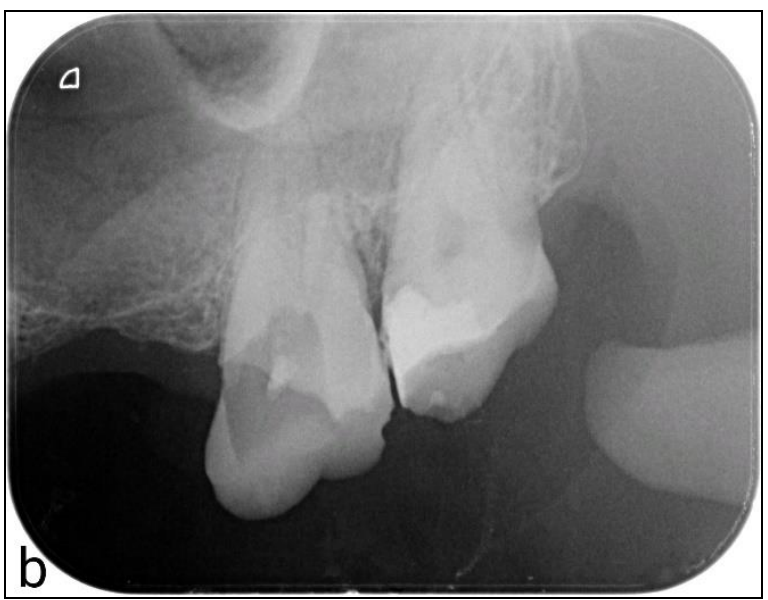

Fig 1b: Temporary restoration and gutta percha removed

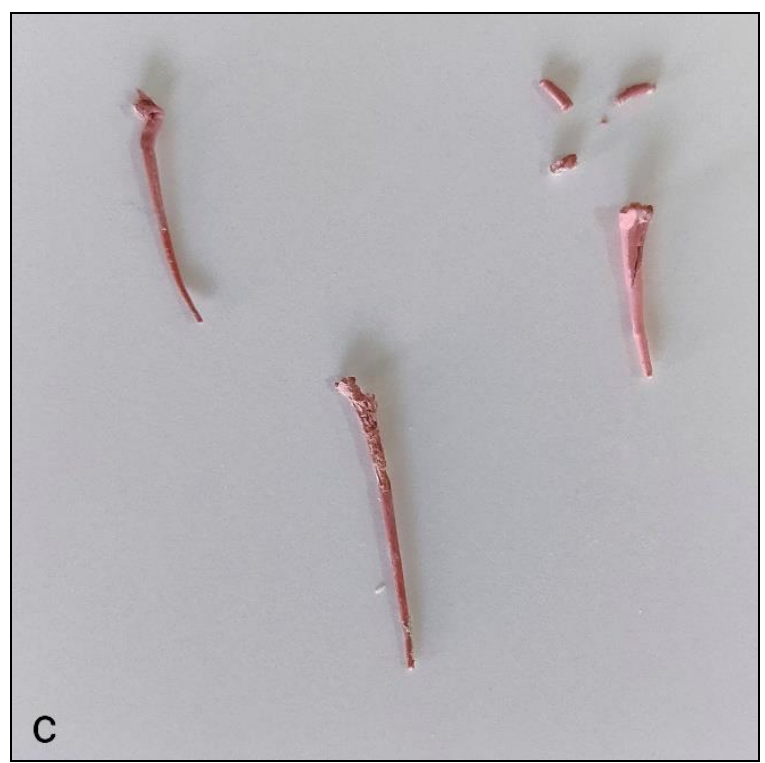

Fig 1c: Clinical photograph of retrieved gutta percha

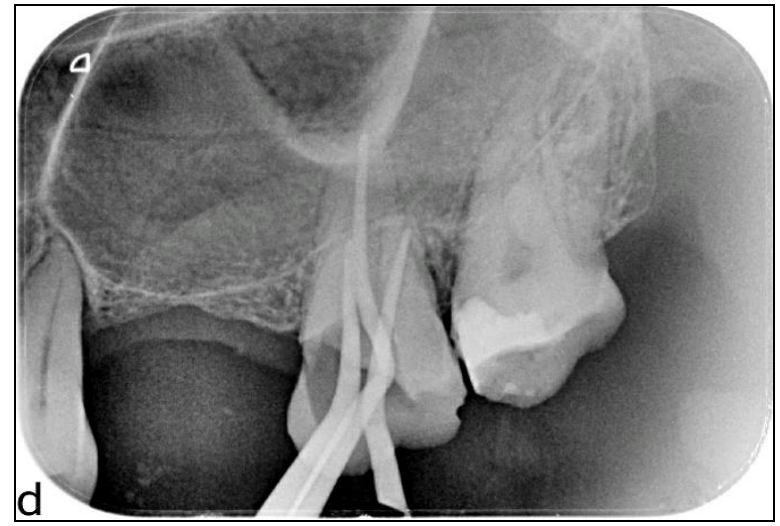

Fig 1d: Master cone radiograph

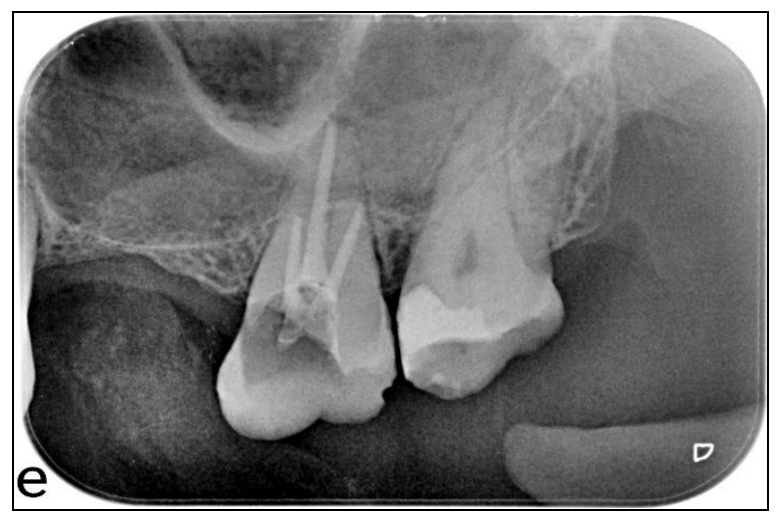

Fig 1e: Post-obturation radiograph

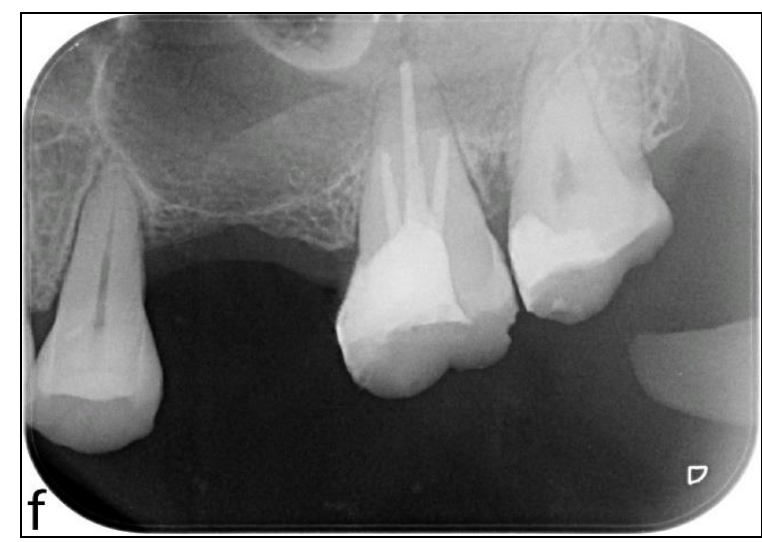

Fig 1f: Post-endodontic restoration (immediate post-op)

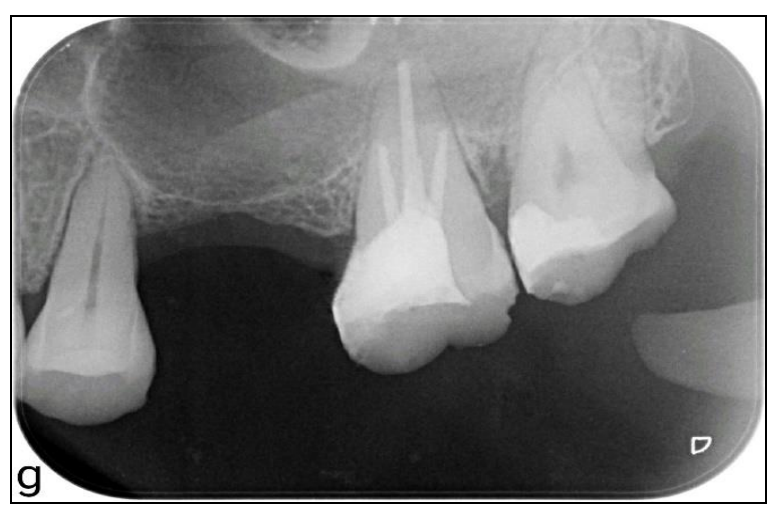

Fig 1g: 3-month follow-up radiograph

\section{Case Report - 2}

A 48-year-old female patient reported to the Department of Conservative Dentistry and Endodontics with a chief complaint of mild pain in the lower right back tooth region in the last 1 month. While recording case history, she revealed 
that she had got an endodontic treatment done in the same region 2 years ago. On clinical examination, exposed root canal filling material was noted in relation to the tooth 45 . On radiographical examination, it was revealed that there was a periapical lesion $2 \mathrm{X} 3 \mathrm{~mm}(\mathrm{PAI}=2)$ in dimensions and inadequate obturation. (Fig 2a) The pain was mild, intermittent and aggravated on chewing food with no history of swelling. The patient had to travel to another city after the treatment. Considering these clinical factors, single-visit endodontic retreatment procedure was initiated for the tooth 45. The temporary restoration removal was followed by removal of gutta percha using Pro-Taper Retreatment files and $\mathrm{H}$-files. (Fig 2b) No chemical solvents were used to remove the gutta-percha or the sealer. The working length was determined after confirming apical patency with \#15 Kfile (Fig 2c), and cleaning and shaping was done using Pro Taper GOLD rotary files, and irrigation was done with $2.5 \%$ sodium hypochlorite activated using Endoactivator. This was followed by use of $17 \%$ EDTA and saline rinse. Final rinse was done using $2 \%$ Chlorhexidine. The master cone IOPA was followed after confirming with the tug back. (Fig 2d) The obturation was done using cold lateral condensation method followed by post-endodontic composite restoration. (Figs 2e, 2f) The 3-month follow-up radiograph (Fig 2g) shows reduction in the size of the lesion, and clinically reduction in the symptoms, both of which indicate successful endodontic retreatment.

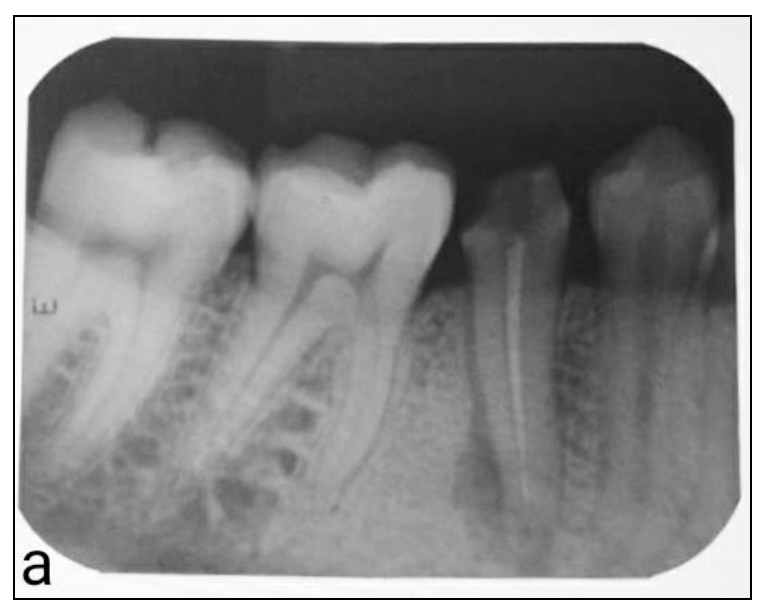

Fig 2a: Pre-operative radiograph (inadequate obturation, $\mathrm{PAI}=2$ )

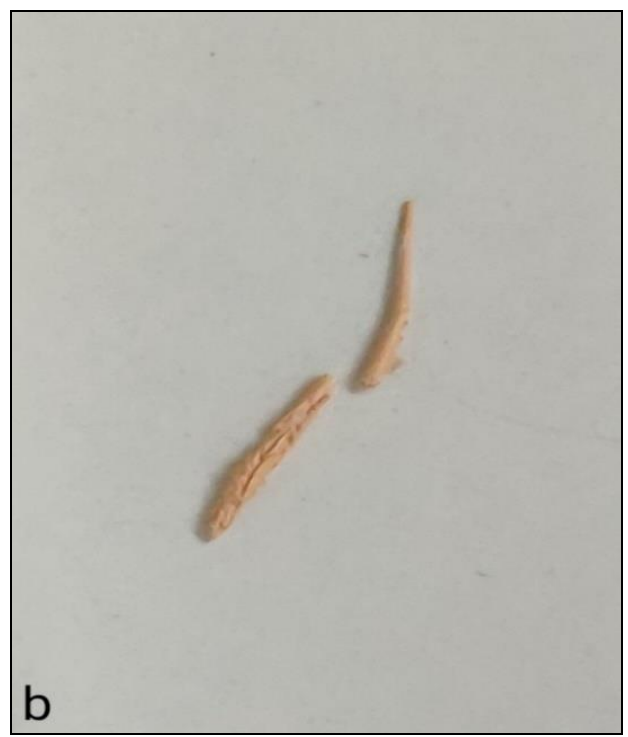

Fig 2b: Clinical photograph of the retrieved gutta percha

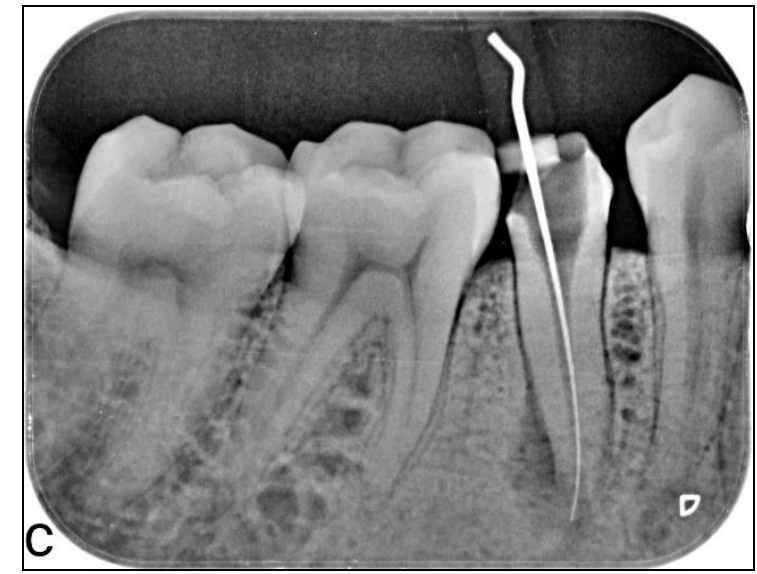

Fig 2c: Working length radiograph

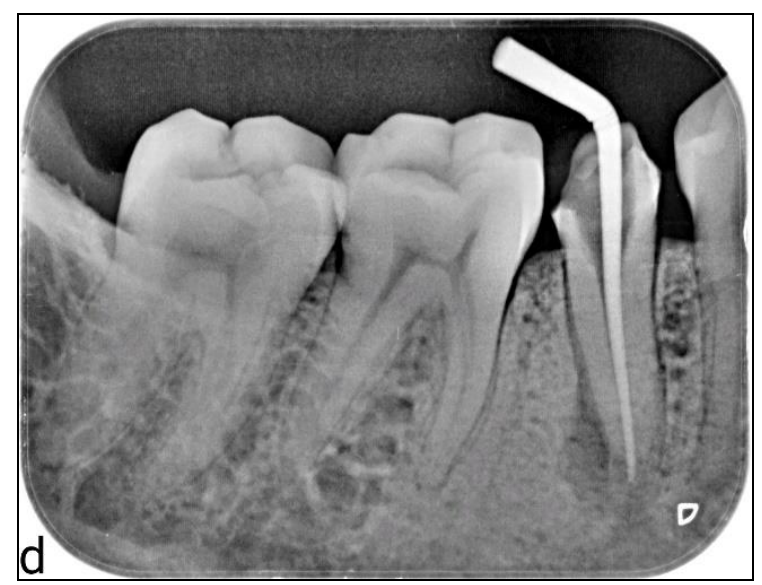

Fig 2d: Master cone radiograph

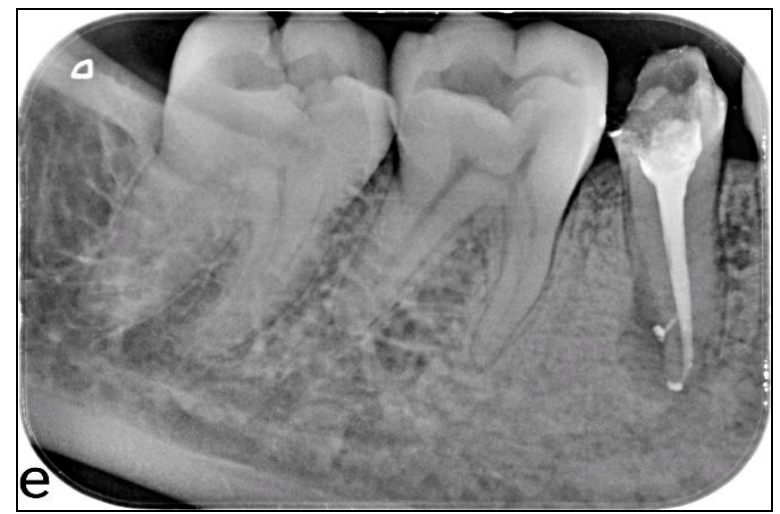

Fig 2e: Post-obturation radiograph

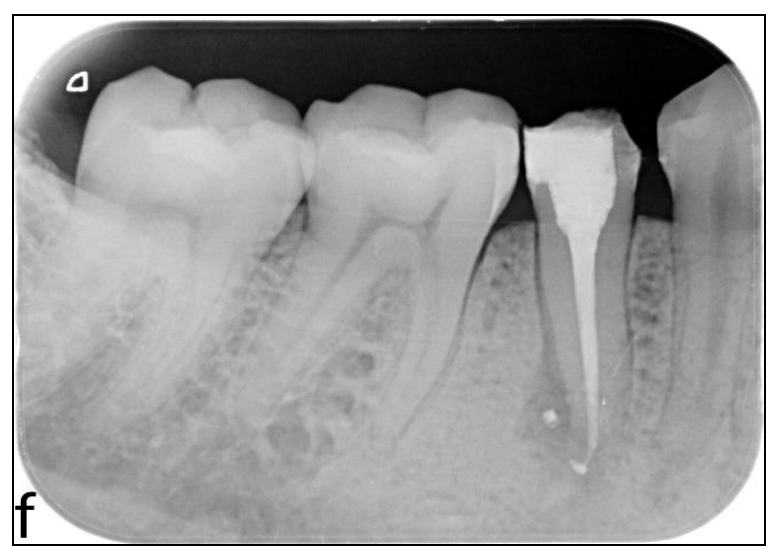

Fig 2f: Post-endodontic restoration (immediate post-op) 


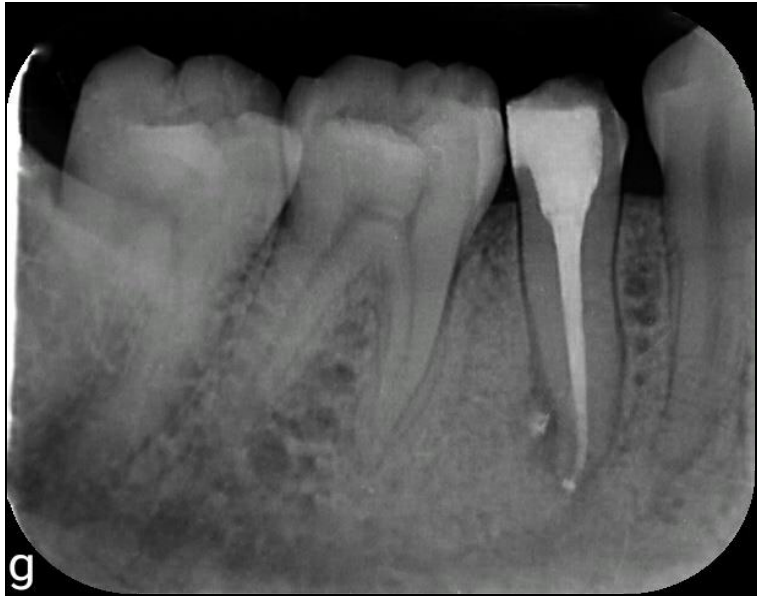

Fig 2g: 3-month follow-up radiograph

\section{Discussion}

Single-visit RCT has been recommended for use in cases with purulent inflammation, traumatic pulpal exposure, or necrotic pulp with a present sinus tract ${ }^{[6]}$. Single-visit RCT is more advantageous than multiple-visit RCT in terms of time and cost. Thus, it is a treatment plan that is more amenable to the needs of busy patients ${ }^{[7,8]}$.

Post treatment diseases could be treated by two options including orthograde retreatment and apical surgery. It is well accepted that nonsurgical retreatment for previously failed endodontic therapy provides favorable results ${ }^{[9]}$. In a previous study, orthograde retreatments presented a success rate of $81 \%$ classified as healed and $93 \%$ as no symptoms and fully functional ${ }^{[10]}$. In a systematic review, it has also been concluded that endodontic surgery offers more favourable initial success but orthograde retreatment yields to a better long-term outcome ${ }^{[11]}$

A retrospective study done by Eyuboglu T.F. et al in 2015 on single visit endodontic retreatments in 173 patients, which presented $90.9 \%$ success rate as $98.2 \%$ of the teeth were asymptomatic and fully functional after a mean observation time of 29 months. Age, gender, tooth type and periodontal defects had no effect on the outcome measures ${ }^{[4]}$. In his study, the criteria for selection of patients was patients having endodontically treated teeth with periapical index score $\leq 2$ $(\mathrm{PAI} \leq 2)$, i.e., periapical lesions which are less than $5 \mathrm{~mm}$ in diameter ${ }^{[4,5]}$.

The irrigation protocol to be followed is not yet established, but in this case series, the highlight was usage of $2.5 \%$ sodium hypochlorite followed by removal of smear layer using $17 \%$ EDTA, which was rinsed using saline, followed by use of $2 \%$ chlorhexidine and a final rinse with saline ${ }^{[12]}$.

Completing the treatment in a single appointment reduces the treatment time and cost, decreases microleakage risk and avoids recontamination of root canals between appointments in multiple-visit treatments. In fact, no significant differences were observed in healing of periapical radiolucency between teeth treated in one visit (without) and those treated in two visits using calcium hydroxide for 4 weeks and that the presence of a positive bacterial culture at the time of filling did not influence the outcome of treatment ${ }^{[5,13]}$.

The type of the definitive restoration was also found to be ineffective on the outcome of orthograde retreatments that was also in agreement with previous studies. The singleappointment approach might also be the reason for high success rate of orthograde retreatments in this study regarding the previous studies ${ }^{[14,15]}$. Patients are being followed up for long-term observations on the outcome measures reported in this study.

Thus, single-appointment root canal retreatments could be considered as a viable treatment option for orthograde retreatment cases with periradicular lesion size smaller than 5 $\mathrm{mm}$ and with mild symptoms.

\section{Conclusion}

Single-visit endodontic treatments and retreatments are being more commonly practiced in the past decade due to the constant evolution in cleaning and shaping dynamics and obturation methods, which is yielding favorable results in patients requiring faster treatments. However, further longterm studies are required to establish the guidelines for single visit endodontic retreatments.

\section{References}

1. Erdem Hepsenoglu Y, Eyuboglu TF, Özcan M. Postoperative Pain Intensity after Single- versus Twovisit Nonsurgical Endodontic Retreatment: A Randomized Clinical Trial. J Endod 2018;44(9):13391346.

2. Yoldas O, Topuz A, Isci AS, Oztunc H. Postoperative pain after endodontic retreatment: single-versus two-visit treatment. Oral Surg Oral Med Oral Pathol Oral Radiol Endod 2004;98:483-7.

3. Gorni FG, Gagliani MM. The outcome of endodontic retreatment: a 2-yr follow-up. J Endod. 2004;30(1):1-4.

4. Eyuboglu TF, Olcay K, Özcan M. A clinical study on single-visit root canal retreatments on consecutive 173 patients: frequency of periapical complications and clinical success rate. Clin Oral Investig 2017;21(5):17611768.

5. Ørstavik D, Pitt Ford TR. Apical periodontitis: microbial infection and host responses. In: Ørstavik D, Pitt Ford TR, eds. Essential endodontology: prevention and treatment of apical periodontitis. Oxford: Blackwell Science, 1998, 1-8.

6. Calhoun RL, Landers RR. One-appointment endodontic therapy: a nationwide survey of endodontists. J Endod 1982;8:35-40.

7. Soltanoff W. A comparative study of the single-visit and the multiple-visit endodontic procedure. J Endod 1978;4:278-81.

8. Jurcak JJ, Bellizzi R, Loushine RJ. Successful single-visit endodontics during Operation Desert Shield. J Endod 1993;19:412-3.

9. Friedman S, Abitbol S, Lawrence HP. Treatment outcome in endodontics: the Toronto Study. Phase 1: initial treatment. J Endod 2003;29(12):787-793.

10. Farzaneh M, Abitbol S, Lawrence HP, Friedman S. Treatment outcome in endodontics: the Toronto Study. Phase II: initial treatment. J Endod 2004;30(5):302-309.

11. Sundqvist G, Figdor D. Life as an endodontic pathogen: ecological differences between the untreated and rootfilled root canals. Endod Topics 2003;6(1):3-28.

12. Basrani, Bettina. Endodontic Irrigation: Chemical Disinfection of the Root Canal System 2015.

13. Van Meerbeek B, Vargas M, Inoue S, Yoshida Y, Peumans M, Lambrechts P et al. Adhesives and cements to promote preservation dentistry. Oper Dent 2001;26(6):119-144.

14. Chugal NM, Clive JM, Spangberg LSW. A prognostic model for assessment of the outcome of endodontic treatment: effect of biologic and diagnostic variables. Oral Surg Oral Med Oral Pathol Oral Radiol Endod 
2001;91(3):342-352.

15. Farzaneh M, Abitbol S, Lawrence HP, Friedman S.

Treatment outcome in endodontics: the Toronto Study.

Phase II: initial treatment. J Endod 2004;30(5):302-309. 